\title{
MEMS AIR-MICROFLUIDIC SENSOR FOR PORTABLE MONITORING OF AIRBORNE PARTICULATES
}

\author{
F.L. Doering ${ }^{1,2}$, I. Paprotny ${ }^{1,3 *}$, and R.M. White ${ }^{1,3 \dagger}$ \\ ${ }^{1}$ Berkeley Sensor \& Actuator Center (BSAC), University of California, Berkeley, USA \\ ${ }^{2}$ The Mechanical Engineering Department, University of California, Berkeley, USA \\ ${ }^{3}$ The Electrical Engineering and Computer Science Department, University of California, Berkeley, USA
}

\begin{abstract}
We present the design, fabrication, and experimental results of a MEMS air-microfluidic sensor for measuring the concentrations of airborne particulate matter (PM) such as tobacco smoke or diesel exhaust. Our sensor $(25 \mathrm{~mm} \times 21 \mathrm{~mm} \times 2 \mathrm{~mm}$ in size) consists of an air-microfluidic circuit that separates the particles by size, then transports and deposits the selected particles onto the surface of a mass-sensitive film bulk acoustic resonator (FBAR). The rate of frequency change of the FBAR due to massloading corresponds to the particle concentration in the sampled air-volume. Our sensors exhibit a low-end detection limit of single $\mu \mathrm{g} / \mathrm{m}^{3}$. The small size of our sensor, combined with high sensitivity, enables it to be used in a portable PM monitor that can record personal PM exposure levels.
\end{abstract}

\section{INTRODUCTION}

Particulate matter (PM) is a category of air-borne pollutant that includes dust, smoke, diesel exhaust and smog. Fine particle (PM 2.5) pollution is especially damaging to our health. Due to their small size these particles penetrate the body's natural filtration systems and lodge deep within the lungs, contribute to several health problems such as reduced lung functionality, bronchitis, and heart attacks. The U.S. Environmental Protection Agency (EPA) has been tightening its safe exposure limit criteria for PM 2.5 [1] and in January of 2012, Beijing Municipal Environmental Protection Bureau started publishing PM 2.5 levels in response to high public demand [2]. While there is increasing demand for PM 2.5 monitoring, the prevailing equipment is fairly bulky and expensive [3], which limits the possibilities for widespread, fine granularity sensing. Portable PM sensors are needed to allow for better identification of pollution sources, health awareness, and pollution control.

In this work we present a microfabricated air-microfluidic PM sensor that has a small form factor so as to be easily integrated into portable personal pollution monitors. The sensor is $25 \mathrm{~mm} \times 21$ $\mathrm{mm} \times 2 \mathrm{~mm}$, almost two orders of magnitude smaller than the smallest currently available commercial devices [4], and an order of magnitude smaller than the macro scale predecessor device from our lab [5]. We describe in detail the principles of operation of the key components our microfluidic circuit; an inertial particle size selector (virtual impactor [6]), as well as particle deposition on the mass-sensing film bulk acoustic resonator (FBAR). We then describe the details of the fabrication process that are used to manufacture prototypes of the PM sensors, and experimental results showing the response of the manufactured prototypes to cigarette smoke and diesel exhaust, two common PM sources. Our results indicate that our sensor has a low-end detection limit of single $\mu \mathrm{g} / \mathrm{m}^{3}$, which is an order of magnitude improvement over the performance of our previous macroscale prototype, and is comparable with large, commercially available PM sensors.

\section{PRINCIPLE OF OPERATION}

A schematic drawing of our air-microfluidic circuit is shown in Figure 1. Air containing suspended particles enters the sensor through the inlet (a). Particles with an aerodynamic diameter larger than $2.5 \mu \mathrm{m}$ are then removed from the air-stream through inertial size-selection (virtual impaction) (b). The remaining particulates (PM 2.5) continue down the microfluidic channels and are deposited onto a mass-sensing element, the exposed surface of an FBAR (c), using an induced temperature gradient across the microfluidic channel (thermophoresis). The concentration of PM 2.5 in the sampled air can be inferred from the rate of the (negative) frequency shift for the mass-loaded FBAR. Negative air pressure at the outlet (d), generated by a stack of commercially available miniature fans $(19 \mathrm{~mm} \times 19 \mathrm{~mm} \times 11 \mathrm{~mm}$ in size $)$, is used to drive the air through the device. (a)

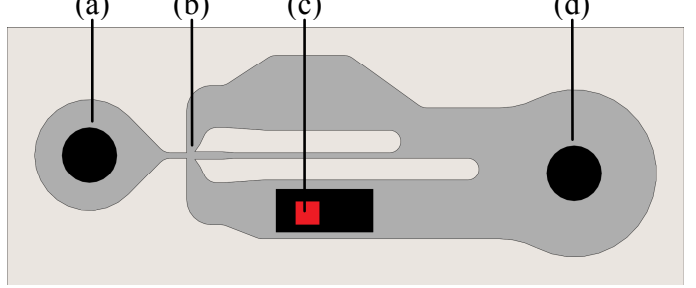

Figure 1: Schematic drawing (top view) of the air-microfluidic channels of our MEMS PM sensor. The channels are shown in dark grey, while the through-wafer holes are in black.

\section{Virtual Impaction (Inertial Size Selectivity)}

The size selectivity of the device is enabled by virtual impaction, where large particles are expelled from the main airstream (major flow) as it is diverted around a tight corner. The momentum of the larger particles cannot be overcome by the drag force of the air, causing these particles to be ejected from the major flow into a minor flow, which exhausts the larger particles. The operation of our virtual impactor is illustrated on the left side of Figure 2, while the right side of Figure 2 shows an SEM of the microfabricated virtual impactor. The inlet jet (a), the major (b) and minor (c) flows are shown.
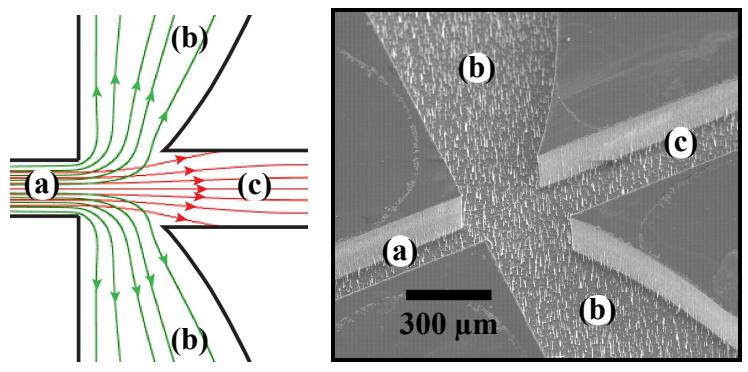

Figure 2: (left) Simulated particle traces for diameters of 5 and 1 $\mu \mathrm{m}$ which both start from the inlet jet (a), but end in the minor flow (c) and major flows (b) respectively. (right) SEM of the microfluidic channels at the size selective region.

The ratio of particles filtered out of the flow increases as their size increases. The aerodynamic diameter at which this ratio is 
one-half is the cutoff size. The cutoff size of an impactor can be described by

$$
d_{50}=\left(\frac{9 \eta W^{2} D\left(S t k_{50}\right)}{\rho_{p} Q}\right)^{1 / 2}
$$

where $d_{50}$ is the particle cutoff diameter, $\eta$ is the dynamic viscosity of air, $W$ and $D$ are the width and depth of the impactor jet respectively, $\rho_{p}$ is the particle density, $Q$ is the jet volumetric flow rate, and $S t k_{50}$ is the stokes number, which is recommended to be 0.59 for rectangular jet impactors [6]. To finely tune the designs of the microfabricated virtual impactor for a cutoff particle diameter of $2.5 \mu \mathrm{m}$, the Finite Element Method (FEM) is used. The simulated collection efficiency (the percentage of particles expelled from the major flow as a function of the particle size) of our virtual impactor is shown in Figure 3.

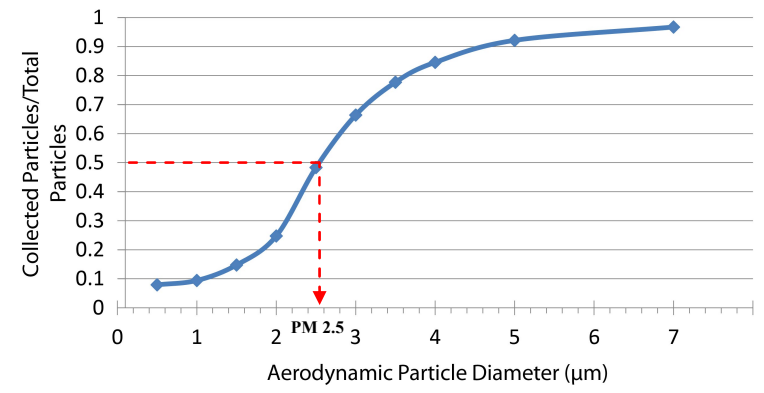

Figure 3: Collection efficiency of simulated particles with aerodynamic diameters near the cutoff diameter for flow through the designed virtual impactor.

Microfabrication techniques allow us to reduce the size of the virtual impactor, which reduces the required airflow and back pressure, lowering the power requirements of the pumping device supplying the negative pressure. To ease fabrication, a rectangular jet impactor is used. A varied channel width is used to affect the viscous pressure loss in each branch of the virtual impactor, thereby regulating the flow rates without valves and the use of only one inlet and one outlet.

\section{Thermophoretic Particle Deposition}

Once the large particles have been removed, a fraction of the remaining particles (PM 2.5) are deposited down-stream from the virtual impactor onto the surface of a mass-sensing FBAR through the process of thermophoresis. Microfabricated heaters create a temperature gradient across the microfluidic channel, which in turn expels the particles from the airflow, and is caused by hot air molecules transferring more momentum to the particle than the cold air molecules. The resulting net thermophoretic force $F_{t h}$ can be approximated by

$$
F_{t h}=\frac{-9 \cdot \pi \cdot d \cdot \eta^{2} \cdot H \cdot \nabla T}{2 \cdot \rho_{g} \cdot T}
$$

where $d$ is the particle diameter, $\eta$ is the dynamic viscosity of the gas, is the temperature gradient, $\rho_{g}$ is the density of the gas, $T$ is the absolute temperature of the gas, and $H$ is the molecular accommodation coefficient defined as

$$
H \cong\left(\frac{1}{1+6 \lambda / d}\right)\left(\frac{k_{a} / k_{p}+4.4 \lambda / d}{1+2 k_{a} / k_{p}+8.8 \lambda / d}\right)
$$

where $k_{a}$ and $k_{p}$ are the thermal conductivities of air and the particle respectively and $\lambda$ is the mean free path length of the gas. The absolute temperature, $T$, is approximated as the average of the temperatures of the heater and the deposition surface, which yields a constant thermal force in the region between the heater and deposition surface. Equating this force with the Stokes drag force yields a thermal particle velocity

$$
V_{t h}=\frac{-3 \cdot \eta \cdot C_{c} \cdot H \cdot \nabla T}{2 \cdot \rho_{g} \cdot T}
$$

where $C_{c}$ is the Cunningham correction factor

$$
C_{c}=1+\frac{2.52 \lambda}{d}
$$

from [6]. The predicted thermal velocity equals $1.36 \mathrm{~mm} / \mathrm{s}$ for our sensor. The deposition rate should be independent of particle size. The thermal gradient is generated by a microfabricated resistive heater as the heat source for the hot side, and a combination of convective and conductive cooling for the cold side. The airstream passing between the heater and the mass sensing element absorbs most of the heat, and the rest is dissipated to the environment by conduction. In order to minimize the power consumed, the heater substrate is quartz, with a low thermal conductivity $(1.3 \mathrm{~W} / \mathrm{K} / \mathrm{m})$. The heaters are released from quartz to further limit the heat transfer.

\section{Mass Sensing}

Our sensor measures the concentration of the particulates by the rate of the frequency shift of the mass-loaded film bulk acoustic resonator (FBAR). As particles are deposited onto the exposed surface of the resonator, the additional mass lowers its resonant frequency. We use FBARs compensated for temperature stability provided by our collaborators at Avago Technologies.

\section{FABRICATION}

Figure 4 shows an exploded CAD drawing of the MEMS PM sensor. The sensor is composed of three wafer die bonded together to form a 3-wafer stack. The top fused quarts wafer serves as a substrate for the thermophoretic heaters as well as the cap for the air-microfluidic circuit. The middle wafer defines the channels of the air-microfluidic circuit, and also contains through-wafer holes for the inlet, outlet, and an insertion opening (a) through which the FBAR is brought into the channel. The bottom fused quartz wafer contains the FBAR (b), its CMOS driver (c), and a polymeric seal (d) for the aforementioned insertion opening.

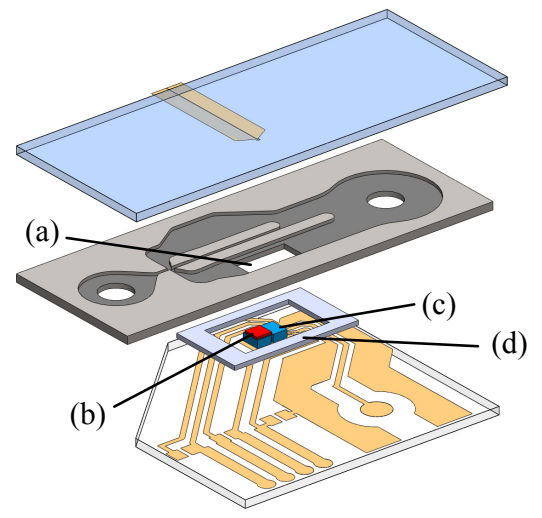

Figure 4: Exploded CAD drawing of the MEMS air-microfluidic PM sensor. 


\section{Top Wafer: Thermophoretic Heaters}

The thermophoretic heaters are fabricated on a fused quartz wafer. The fabrication process is illustrated in Figure 5. First, $\mathrm{a} \approx 2$ $\mu \mathrm{m}$ layer of in-situ doped polysilicon is deposited through an LPCVD process $\left(50 \% \mathrm{SiH} 4 / 50 \% \mathrm{PH} 3,615^{\circ} \mathrm{C}\right)$. In order to avoid the cracking of the quartz wafer, the polysilicon is not annealed. However, the un-annealed polysilicon layer has a sheet resistance of $\approx 40 \mathrm{ohm} / \square$, which is sufficient for our application. A $100 \mathrm{~nm}$ layer of $\mathrm{Cr} / \mathrm{Au}$ is then evaporated using an E-beam evaporator. The $\mathrm{Au} / \mathrm{Cr}$ layer is then lithographically patterned using an iodinebased gold etchant and CR-7, defining the high conductivity leads for the thermophoretic heaters. The doped polysilicon layer is then etched by a dry RIE process $\left(90 \% \mathrm{SF}_{6} / 10 \% \mathrm{O}_{2}, 66 \mathrm{sccm}\right)$, defining the $8 \mu \mathrm{m}$ wide resistive heater elements. The wafer is diced into individual die, and the heaters are released from the quarts wafer by a 10 -minute etch in $49 \% \mathrm{HF}$. An optical micrograph of a fabricated thermophoretic heater, taken through the top quartz wafer is shown in Figure 6. Note the dark area (a) representing the underetched region of the polysilicon layer.

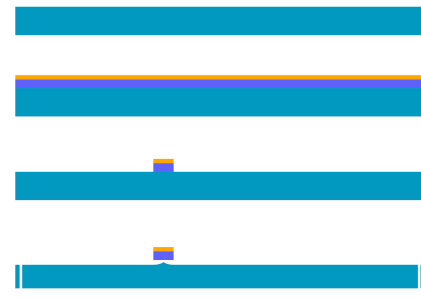

\section{Quartz Wafer LPCVD $2 \mu \mathrm{m}$ PolySi (p-type) $100 \mathrm{~nm} \mathrm{Cr/Au}$ \\ Define heaters, RIE PolySi etch Wet-etch $\mathrm{Cr} / \mathrm{Au}$ \\ Sub-dice \\ Underetch heaters in $\mathrm{HF}$}

Figure 5: Fabrication process for the top wafer, defining the thermophoretic heaters.

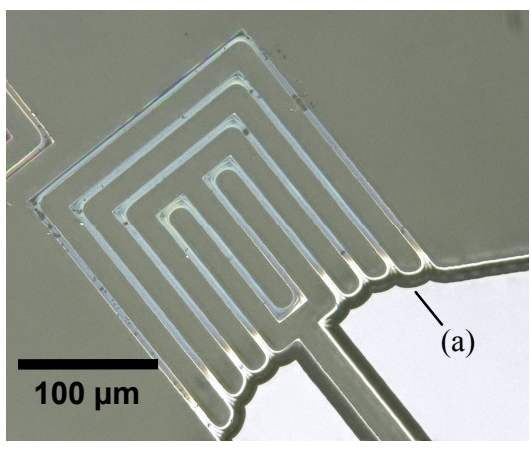

Figure 6: Optical micrograph of a finished heater taken through the quartz wafer.

\section{Middle Wafer: Micro-Fluidic Channels}

The middle wafer defines the microfluidic channels of the MEMS PM sensor. The fabrication process for the middle wafer is shown in Figure 7. The microfluidic channels are lithographically defined using a DRIE process $\left(\mathrm{C}_{4} \mathrm{~F}_{8}\right.$ passivation, $90 \% \mathrm{SF}_{6} / 10 \% \mathrm{O}_{2}$ RIE etch) to etch $200 \mu \mathrm{m}$ deep channels, as well as the throughwafer holes for the inlet, outlet, and FBAR insertion opening. The wafer is then diced into individual die.

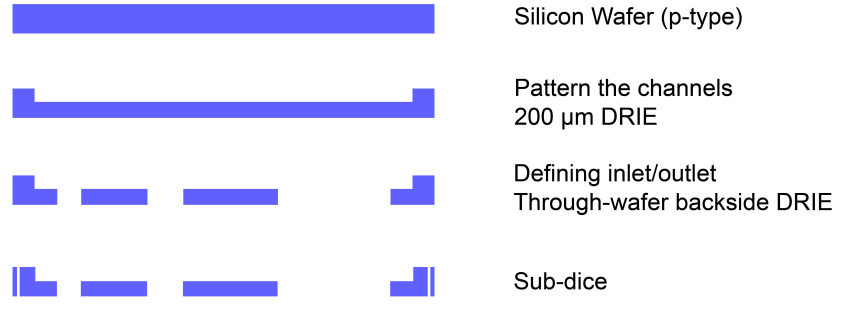

Figure 7: Fabrication process for the middle wafer, defining the channels of the microfluidic circuit.

\section{Bottom Wafer: The FBAR Substrate}

The bottom fused quartz wafer contains the FBAR, its CMOS driver, the gold connection traces, as well as a polymeric spacer seal. The fabrication process for this wafer is outlined in Figure 8. First, $100 \mathrm{~nm}$ of $\mathrm{Cr} / \mathrm{Au}$ is evaporated onto the wafer, and then is lithographically patterned to define the power and signal traces for the driver CMOS. The wafer is then diced. A $475 \mu \mathrm{m}$ tall $1 \mathrm{~mm} \times$ $1 \mathrm{~mm}$ pedestal is attached using cyanoacrylate adhesive at the FBAR location, to ensure the insertion of the FBAR inside the microfluidic channel. The driver CMOS die (a Pierce oscillator) and the FBAR die are then physically bonded to the wafer and the pedestal using the same cyanoacrylate adhesive. The FBAR is then wire bonded to the CMOS, and the CMOS is wire-bonded to the gold traces. Power and RF signal lead are soldered onto the traces. Finally, a $285 \mu \mathrm{m}$ adhesive polymeric seal (made out of a triple stack of dicing tape) is applied around the sensing elements to act as a seal for the insertion opening and to set the distance that the FBAR protrudes into the microfluidic channel.

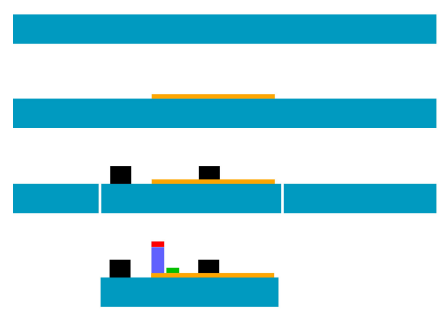

Quartz Wafer

$100 \mathrm{~nm} \mathrm{Cr} / \mathrm{Au}$

Define electrodes by wet-etch

Attach the $285 \mu \mathrm{m}$ seal Sub-dice

Attach FBAR on $475 \mu \mathrm{m}$ pedestal Attach CMOS driver, wirebond

Figure 8: The fabrication process for the bottom wafer, which serves as the substrate for the mass-sensitive FBAR and CMOS driver.

\section{Final Assembly}

Figure 9 illustrates the bonding process of the three wafers. First, a top wafer die is bonded to a matching middle wafer die to form the air-microfluidic circuit, while the heaters are aligned above the insertion opening. To ensure a good seal around the small features of the microfluidic channels, we used adhesive bonding by applying dispenser-printed epoxy (72\% Epon 830 resin, 28\% Epikure 3370 curing agent), and curing overnight on a hotplate at $60^{\circ} \mathrm{C}$. The same epoxy mixture is applied to the polymeric seal, and the bottom wafer die is visually aligned and attached to the top/middle wafer stack, placing the FBAR into the microfluidic channel through the insertion opening. The spacing between the surface of the FBAR and the heater is designed for a nominal spacing of $100 \mu \mathrm{m}$. The sensor is sealed with silicone (Osi Sealants/Henkel Adhesives) around the edges of the bonded die to prevent leaks and increase the structural stability of the final device. 


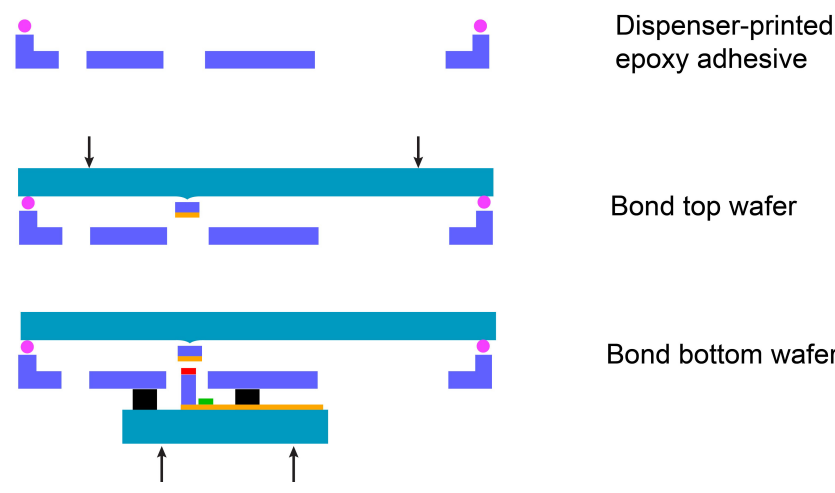

Figure 9: Bonding of the three wafers to complete the assembly of the MEMS PM sensor.

Figure 10 shows a fabricated MEMS PM sensor prototype; labels (a) and (b) correspond to the size-selection and deposition areas respectively. The alignment of all the layers can be seen in the optical micrograph of the sensing area, taken through the top quartz wafer and shown in Figure 11. Two FBARs are visible as pink pentagons, partially obscured by the two heaters. Bond wires connect the CMOS oscillator circuit to one of the FBARs and to the bottom wafer (out of focus).

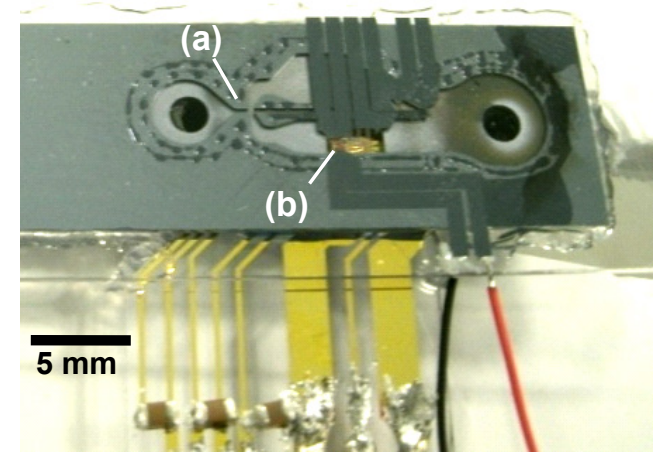

Figure 10: Photograph of a complete prototype of the MEMS airmicrofluidic PM sensor.

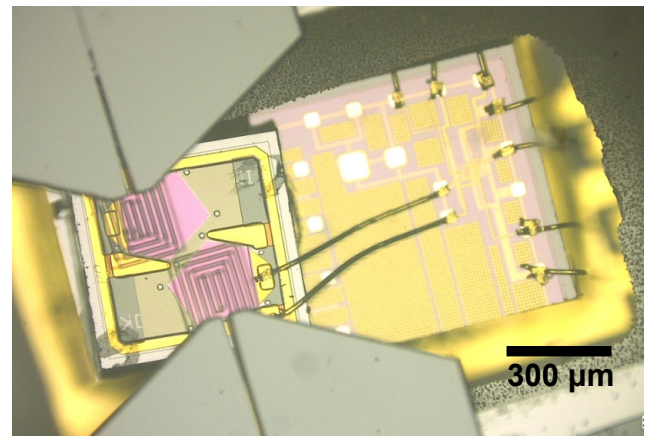

Figure 11: Optical micrograph of the sensing area in an assembled prototype of the MEMS PM sensor (labeled as (b) in Figure 10).

\section{EXPERIMENTAL RESULTS}

Experimental Setup

Two fabricated MEMS PM sensor prototypes (labeled A and B) were tested in an air-quality testing chamber $\left(25 \mathrm{~m}^{3}\right)$ at the Environmental Energy Technologies Division (EETD) of Lawrence Berkeley National Lab. To generate a representative concentration of tobacco smoke, a single cigarette was lit and smoked using a custom built machine, simulating a single cigarette being smoked in a closed room. To generate the diesel exhaust, a portable diesel generator was used. A HEPA filtration unit (Control Resources Systems Inc. 600L) was used to lower the concentration of particles during testing. A DustTrak ${ }^{\mathrm{TM}}$ Aerosol Monitor 8520, calibrated based on [7] and gravimetric analysis [6], was used to provide a reference for the particulate concentration in the test chamber. The resonant frequency of the FBAR was measured using a spectrum analyzer (Agilent 8562EC), and was recorded and tracked from a laptop using a custom-developed LABVIEW (v. 10.0f2) interface.

\section{Results}

The response of the MEMS PM sensor to tobacco smoke and diesel exhaust, during two representative experiments is shown on Figures 12 and 13, respectively. In both cases, good correlation can be observed between our device and the DustTrak reference measurements. The steps in the graphs are the result of periodic activation of the HEPA filtration unit, which causes a rapid drop in $\mathrm{PM}$ concentration.

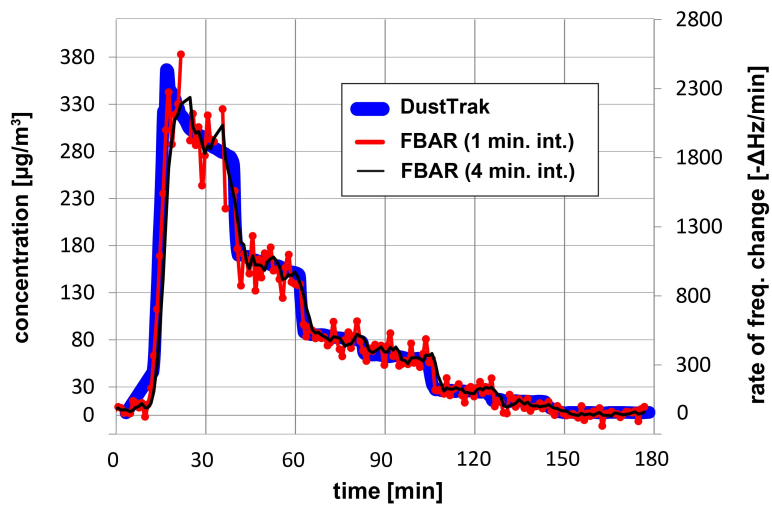

Figure 12: Response of the sensor (prototype A) to cigarette smoke during a representative experiment.

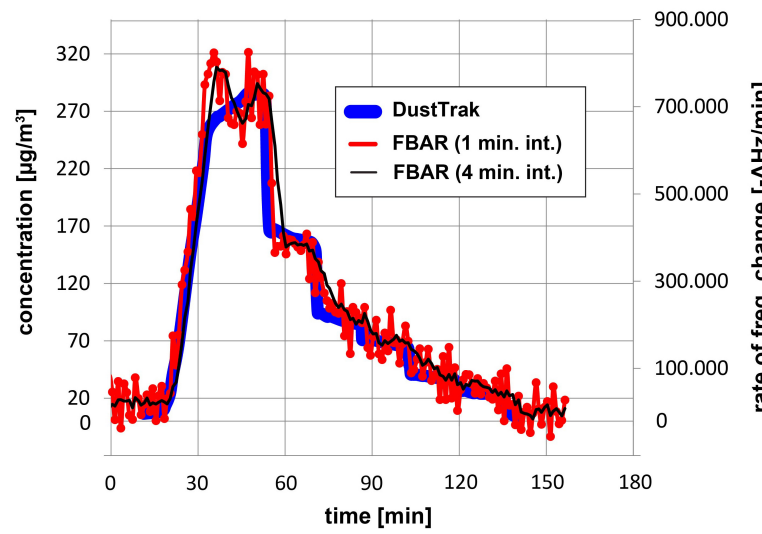

Figure 13: Response of the sensor (prototype B) to diesel exhaust during a representative experiment.

Table I shows the sensitivity coefficient, relating frequency 
shift rate to concentration, for both prototypes. Prototype A failed before the diesel exhaust test was performed; hence sensitivity coefficient for diesel exhaust for that prototype is unavailable. Note the drop in tobacco smoke concentration from 25 to $15 \mu \mathrm{g} / \mathrm{m}^{3}$ around min. 130 in Figure 12. This step is apparent in the $4 \mathrm{~min}$. integration time sensor plot, suggesting a low-end detection limit (with $4 \mathrm{~min}$. integration time) below $10 \mu \mathrm{g} / \mathrm{m}^{3}$ (for prototype A).

Table 1: Average sensitivity coefficient (in $\mathrm{Hz} / \mathrm{s}$ per $\mathrm{mg} / \mathrm{m}^{3}$ ) for sensor prototypes $A$ and $B$ for tobacco smoke and diesel exhaust. Standard deviation is shown in parenthesis.

\begin{tabular}{|l|c|c|}
\hline & $\begin{array}{c}\text { Tobacco } \\
\text { Smoke }\end{array}$ & Diesel Exhaust \\
\hline Prototype A & $96(11.31)$ & NA \\
\hline Prototype B & $28.3(3.82)$ & $40.93(3.76)$ \\
\hline
\end{tabular}

Based on estimated sensor variability, which at low PM concentrations is approximately $50 \mathrm{~Hz}$, we estimate a low-end detection limit of approximately $2 \mu \mathrm{g} / \mathrm{m}^{3}$ with $10 \mathrm{~min}$. integration time (for prototype A).

The sensitivity coefficient of both prototypes is strongly dependent on the rate of the airflow through the microfluidic channel (data in Table 1 is taken at highest flow rate). Such strong dependency indicates that our sensor is depleting the particles in the microfluidic channel above the FBAR, and hence reducing the available particle mass at lower flow rates. This is confirmed by the deposition pattern observed in an optical micrograph of the FBAR after several experimental runs, shown in Figure 14. The air flows from the right (indicated by black arrow). The deposited particles can be observed as a grey fuzzy crescent (outlined by a striped line). The crescent shape, as opposed to an oval, indicates that there are significantly more particles deposited on the upstream half of the heater than the downstream half. This nonuniformity indicates that the particles have been nearly depleted by the time the airflow passes under the right side of the heater.

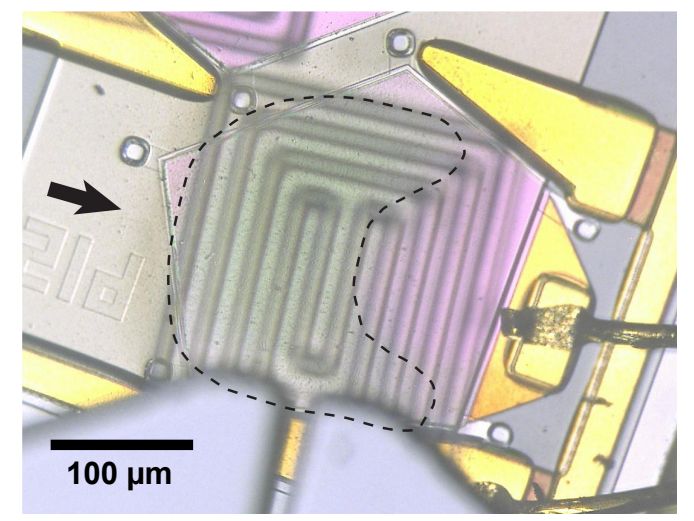

Figure 14: Optical micrograph of the FBAR viewed through the quartz wafer and silicon heater after several deposition runs.

Both tobacco smoke and diesel exhaust contain particles that are much smaller than the cut-off particle diameter of our virtual impactor, preventing us from validating its collection efficiency in our current experimental setup; we leave this validation for future work.

\section{CONCLUSION}

In this paper, we presented the designs, fabrication, and experimental results of an air-microfluidic MEMS PM sensor. The sensor is two orders of magnitude smaller than commercially available PM sensors and an order of magnitude smaller than the previously presented macro-scale prototype by our group. The single $\mu \mathrm{g} / \mathrm{m}^{3}$ low-end detection limit of our sensor, combined with its small size enables our device to be used as a portable, perhaps wearable, personalized PM exposure monitor, which is sufficiently sensitive to monitor compliance with the EPA air quality standard. The use of microfabrication techniques enables us to create complex air-microfluidic circuits with a small footprint. This airmicrofluidic lab-on-a-chip will enable comprehensive and portable air quality monitoring.

The depletion of particulates from the airstream during deposition indicates that we can further increase the low end detection limit by increasing the air flow rate through the microfluidic channel. We will investigate this possibility in subsequent testing.

In this work, we recorded the signal from our sensor using a spectrum analyzer. We are currently working with several collaborators to develop the frequency-tracking electronics and connect our sensor to a cellular radio, enabling massively distributed portable PM sensing applications.

\section{ACKNOWLEDGEMENTS}

The authors gratefully acknowledge Intel Corp. for their generous financial support of this work. The authors would also like to thank Lara Gundel and Dough Sullivan from EETD for their help in testing the sensor prototypes, Allison Woodruff and Alan Mainwaring from Intel Corp., as well as Paul Solomon from the Environmental Protection Agency for their comments and technical discussion. Finally, we would like to thank Rich Ruby and Avago Technologies for providing the FBARs.

\section{REFERENCES}

[1] "Particulate Matter," The United States Environmental Protection Agency (EPA), http://www.epa.gov/oar/particlepollution/, Downloaded on 3/31/12.

[2] "Beijing Releases PM 2.5 Air Quality Readings," Caixin Online, http://english.caixin.com/2012-0121/100350762.html, Downloaded on 3/31/12.

[3] "Met One Aerocet 531 Mass/ Laser Particle Counter," BPA Air Quality Solutions LLC, http://www.breathepureair.com/met_one_gt531.html, downloaded on 3/31/12.

[4] “DUSTTRAK II Aerosol Monitor 8532," TSI Incorporated, http://www.tsi.com/DUSTTRAK-II-Aerosol-Monitor-8532/, Downloaded on 3/31/12.

[5] J. Black, A. Elium, R. White, M. Apte, L. Gundel, and R. Cambie, "MEMS-Enabled Miniaturized Particulate Matter Monitor Employing 1.6 GHz Aluminum Nitride Thin-Film Bulk Acoustic Wave Resonator (FBAR) and Thermophoretic Precipitator," Ultrasonics Symposium, 2007, 10/28-31/07, IEEE, (2007), pp. 476 - 479.

[6] W. C. Hinds, Aerosol Technology: Properties, Behavior, and Measurement of Airborne Particles, 2nd Ed., John Wiley \& Sons, New York, 1999.

[7] S. Liu and Y. Zhu, "A case study of exposure to ultrafine particles from secondhand tobacco smoke in an automobile", Indoor Air, 20: 412-423 (2010).

\section{CONTACT}

* I. Paprotny, igorpapa@eecs.berkeley.edu

† R.M. White, tel: +1-510-642-0540; rwhite@eecs.berkeley.edu 